\title{
Recognition of Facial Expressions using Local Mean Binary Pattern
}

\author{
Mahesh Goyani* and Narendra Patel ${ }^{+}$ \\ * Department of Computer Engineering, Charotar University of Science and Technology, Changa, India \\ + Department of Computer Engineering , BVM Engineering College, V.V.Nagar, India
}

Received 12 $2^{\text {th }}$ Feb 2017; accepted 22 $2^{\text {nd }}$ May 2017

\begin{abstract}
In this paper, we propose a novel appearance based local feature descriptor called Local Mean Binary Pattern (LMBP) for facial expression recognition. It efficiently encodes the local texture and global shape of the face. LMBP code of a pixel is produced by weighting the thresholded neighbor intensity values with respect to mean of $3 \times 3$ patch. LMBP produces highly discriminative code compared to other state of the art methods. The micro pattern is derived by thesholding on mean of the patch, and hence it is robust against illumination and noise variations. An image is divided into $\mathrm{M} \times \mathrm{N}$ regions and feature descriptor is derived by concatenating LMBP distribution of each region. We also propose a novel template matching strategy called Histogram Normalized Absolute Difference (HNAD) for comparing LMBP histograms. Rigorous experiments prove the effectiveness and robustness of LMBP operator. Experiments also prove the superiority of HNAD measure over well-known template matching methods such as L2 norm and Chi-Square. We also investigated LMBP for expression recognition in low resolution. The performance of the proposed approach is tested on well-known datasets CK, JAFFE, and TFEID.
\end{abstract}

Key Words: Local Binary Pattern, Local Direction Pattern, Local Mean Binary Pattern, Histogram Normalized Absolute Difference, Support Vector Machine.

\section{Introduction}

Facial expression plays a vital role in communicating emotions and intentions [1]. Visual clues and information lead towards a better understanding in a conversation. Application area of FER covers a broad spectrum, including grading of physical pain, smile detection [2], [3], [4], driver fatigue detection [5], patient pain assessment [6], video indexing, robotics and virtual reality [7], depression detection [8] etc. Mental state is imitated on the face in the form of various expressions; affective computing models them in an appropriate computer actions [9]. Interpretation of facial expression through the machine can become a driving force for the future automation interfaces such as car driving, robotics, driver alert systems, human-computer interfaces etc. [7], [10].

Investigation of the Physiognomy and facial expression dates to the era of Aristotle (4 ${ }^{\text {th }}$ century). Physiognomy is the Greek word, in which physis means "nature" and gnom means "judge". It corresponds to judging people's character from their external appearance, especially from the face [11]. In 1872, the first

Correspondence to: <mgoyani@gmail.com>

Recommended for acceptance by $<$ Angel Sappa $>$

http://dx.doi.org/10.5565/rev/elcvia. 1058

ELCVIA ISSN: 1577-5097

Published by Computer Vision Center / Universitat Autonoma de Barcelona, Barcelona, Spain 
preliminary experiment on facial expression was conducted by Charles Darvin, that had a direct influence on modern FER research during that time. He mentioned the generality of facial expressions across human and animals in his well-known book "The Expression of the Emotions in Man and Animal" [12]. He observed how humans and animals exhibit the common characteristics while expressing their emotions. Both have a tendency of showing their ocular muscles and tighten their teeth when they are in anger state. Darvin's claim of universality in expression was reinforced by the series of cross cultural experiments conducted by Ekman and Friesen in 1971 [13]. They postulated a range of expressions into six judgmental classes, which are anger, disgust, fear, happy, sad and surprise. Most of the research on facial expression is centered around detecting these basic six expressions.

In this paper, we propose a novel appearance based local face descriptor called Local Mean Binary Pattern. LMBP code is obtained by deriving LBP code of mean centered patch of size $3 \times 3$. Consequently, this approach is robust against non-monotonic gray level change and random noise. LMBP efficiently characterizes the local intensity variation and structural information. We also present a novel template matching technique called Histogram Normalized Absolute Difference (HNAD) for matching the LMBP histograms. Proposed measure normalizes the absolute difference of two histogram values by the union of histograms. Experiments show that HNAD outperforms the customary template matching techniques like L2 norm and Chi-square. The discriminative capability of LMBP is also investigated for low-resolution facial expression recognition. Results show the stability of over a wide range of low-resolution images, which motivates the use of LMBP for real world applications.

Rest of the paper is organized as follows: State of the art methods have been studied in section 2. A brief review on local statistical operators like Local Binary Pattern (LBP) and Local Directional Pattern (LDP) is presented in Section 3. The proposed appearance based operator Local Mean Binary Pattern is introduced in Section 4. Section 5 describes the novel template matcher HNAD along with experimental setup and facial expression datasets. Optimal parameter selection, result analysis and expression recognition in low resolution are presented in section 6. Finally, the work is summarized in Section 7.

\section{State of the Art Methods}

There are commonly two approaches for facial feature extraction: geometric feature based methods and appearance feature based methods [7], [9], [14], [15]. Many modern facial expression recognition systems utilize the vector of position and relation of certain facial key points as a feature descriptor. Such systems are referred as geometry based systems. The facial key points, whose position is localized are known as fiducial points. Typically, fiducial points are positioned along the nose, lip, eye and eyebrow. The motivation for using geometry based systems is that each expression affects the relative position of such fiducial points. Appearance or texture based analysis of the image is very useful in applications at different levels [16]. The texture is the visual property which characterizes the surface by means of variations in shape, illumination, reflectance, shadow, absorption etc. In appearance-based methods, certain filters or kernel functions are applied on the face to extract a feature vector. Appearance/texture features are more suitable for capturing subtle appearance changes (e.g. wrinkles) of the face, while geometric features are more capable of representing the shape and location information of facial components (e.g. mouth, eye, nose etc.). Geometry and appearance based features are depicted in Figure 1.
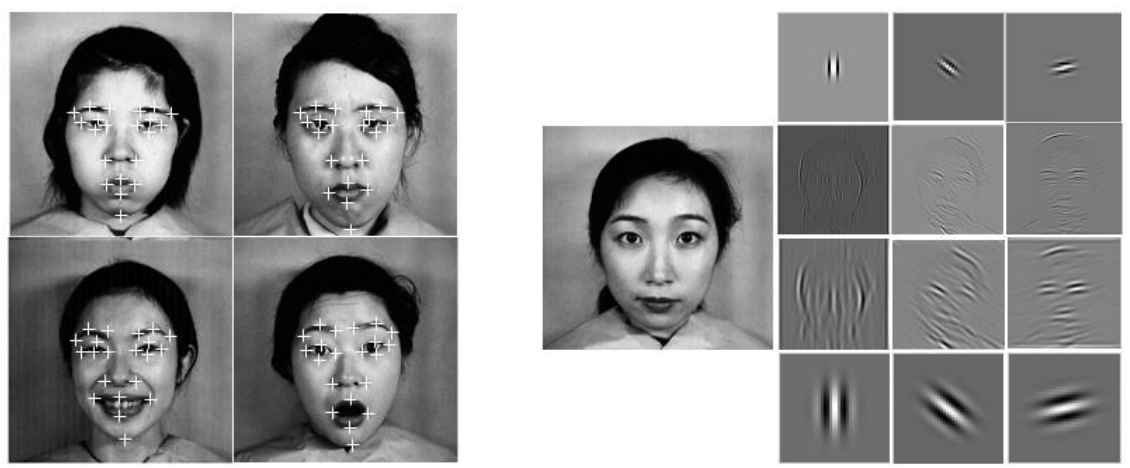

Fig. 1. Illustration of geometric (left) and appearance (right) features 
Based on the region of operation, facial extraction methods are further classified into global and local. Global methods operate on entire image and hence they are also known as holistic methods. Optical flow [17], [18], Eigenface [19], Fisherface [20], Independent Component Analysis (ICA) [21] are few of the widely used holistic methods. Even though these methods have been significantly used and explored, local descriptors have gained the attention of researchers because of their robustness and invariance property. Local feature extraction methods operate on a small neighbourhood of the pixel. Feature descriptor is obtained by collecting features computed locally. Local Binary Pattern (LBP) [22], multi-resolution LBP [23], Local Directional Pattern (LDP) [24], Gabor filter [25], Local Gabor Binary Pattern (LGBP) [10] are some of the well-studied local feature extraction methods.

Comprehensive and widely cited surveys by Samal and Lyengar [26], Pantic and Rothkrantz [7] and Fasel and Luetin (2003) [8] are available that perform an in-depth study of the published work from 1990 to 2001. Recent advancements in the $21^{\text {st }}$ century are surveyed by Tian et al. [27], Zeng et al. [8], Dailey et al. [28], Murtaza et al. [29] and Corneanu et al. [14].

\section{$3 \quad$ Revisiting Local Statistical Operators}

The two important aspects of facial expression recognition comprise facial representation and design of the classifier. Facial representation refers to finding a set of features that can effectively characterize the face image. The optimal feature should maximize the intra-class similarity and minimize the interclass similarity [30]. The success of an appropriate classifier ultimately depends on an effective facial representation, which can lead to better recognition rate.

\subsection{Local Binary Pattern}

Local binary pattern is comprehensively studied powerful texture analysis descriptor. In 1996, Ojala et al. [22] proposed LBP for analysing macro and micro patterns of the image. It encodes micro patterns in the local neighbourhood of the pixel using knowledge of neighbour pixel intensity. LBP is invariant to changes in monotonic gray level. LBP code is generated by weighting the thresholded P neighbours by the decimal values. LBP code for the center pixel $\left(\mathrm{x}_{\mathrm{c}}, \mathrm{y}_{\mathrm{c}}\right)$ is computed as,

$$
L B P_{P, R}\left(x_{c}, y_{c}\right)=\sum_{n=0}^{P-1} 2^{n} * S\left(i_{n}-i_{c}\right), \quad S(x)= \begin{cases}1, & x \geq 0 \\ 0, & x<0\end{cases}
$$

Where, $i_{n}$ denotes the gray value of equally spread out $\mathrm{P}$ neighbours on the perimeter of radius $\mathrm{R}$ with respect to center $\left(\mathrm{x}_{\mathrm{c}}, \mathrm{y}_{\mathrm{c}}\right)$, and $i_{c}$ is the gray value of center pixel. Binary value assigned by function $\mathrm{S}($.$) over$ $\mathrm{P}$ bits is weighted by the decimal value, which gives the LBP code for center pixel. Figure 2 illustrates the steps for LBP code generation.

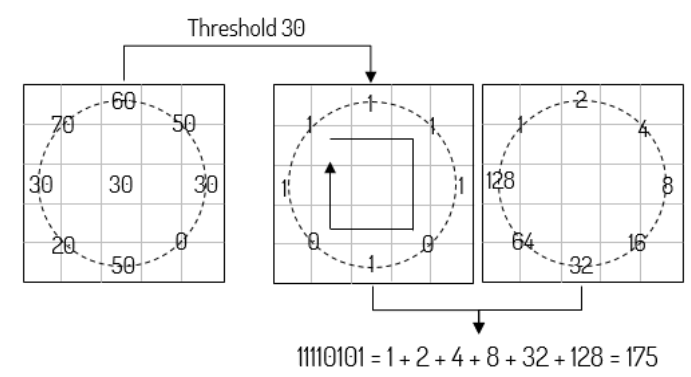

Fig. 2. LBP operator to compute the LBP code for $\mathrm{P}$ bits and radius $\mathrm{R}$

Many variants of LBP are proposed to make it more robust. Ojala et al. [31] introduced uniform binary pattern to reduce the feature dimensions. By combining Gabor filtering with LBP, Local Gabor Binary Patterns (LGBP) [32] was proposed to extend LBP to multiple resolutions and orientations. Shan et al. [30] presented boosted LBP based approach to extract most discriminative LBP features. They also extend the experiment for low-resolution images. To further reduce the computation, Hablani et al. [33] employed LBP on prominent regions of the face. LBP histograms of eyes, mouth, nose are concatenated to form the final feature vector.

\subsection{Local Directional Pattern}


Jabid et al. [24] present a Local Direction Pattern (LDP) descriptor for facial representation. LDP derives 8-bit binary pattern by encoding edge response value in 8 directions using Kirsch edge detector. Kirsch detects directional response more accurately compared to Prewitt or Sobel edge detectors. Though Kirsch operator is robust, it is computationally demanding as it uses eight different masks to compute response in each direction. Masks used to compute LDP are shown in Figure 3.

$$
\begin{aligned}
& {\left[\begin{array}{ccc}
5 & 5 & -3 \\
5 & 0 & -3 \\
-3 & -3 & -3
\end{array}\right]} \\
& \text { North West } \mathrm{M}_{0} \\
& {\left[\begin{array}{ccc}
-3 & -3 & -3 \\
-3 & 0 & 5 \\
-3 & 5 & 5
\end{array}\right]}
\end{aligned}
$$

South East $\mathrm{M}_{4}$

$$
\begin{aligned}
& {\left[\begin{array}{ccc}
5 & 5 & 5 \\
-3 & 0 & -3 \\
-3 & -3 & -3
\end{array}\right]} \\
& \text { North } M_{1} \\
& {\left[\begin{array}{ccc}
-3 & -3 & -3 \\
-3 & 0 & -3 \\
5 & 5 & 5
\end{array}\right]}
\end{aligned}
$$

South $\mathrm{M}_{5}$ $\left[\begin{array}{ccc}-3 & 5 & 5 \\ -3 & 0 & 5 \\ -3 & -3 & -3\end{array}\right]$
North East $\mathrm{M}_{2}$
$\left[\begin{array}{ccc}-3 & -3 & -3 \\ 5 & 0 & -3 \\ 5 & 5 & -3\end{array}\right]$

South West $\mathrm{M}_{6}$ $\left[\begin{array}{ccc}-3 & -3 & 5 \\ -3 & 0 & 5 \\ -3 & -3 & 5\end{array}\right]$
East $M_{3}$
$\left[\begin{array}{ccc}5 & -3 & -3 \\ 5 & 0 & -3 \\ 5 & -3 & -3\end{array}\right]$

West $\mathrm{M}_{7}$

Fig. 3. Kirsch edge detector masks

Mi-th mask generates mi response, where $i=0,1, \ldots, 7$. The response of eight masks is collected in a $3 \times 3$ matrix as displayed in Figure 4. Neighbor responses are thresholded with respect to mk-th directional response. The value of $\mathrm{k}$ is chosen by rigorous experiments. LBP of these responses gives the LDP code for center pixel. Histogram of these LDP values is used as a feature descriptor.

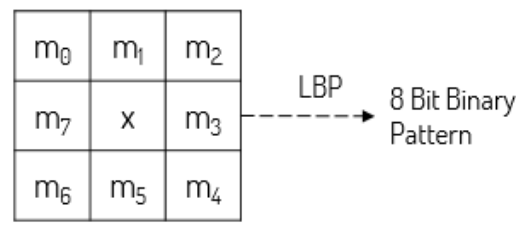

Fig. 4. Generation of LDP code

Given the center pixel and 8-Kirsch masks, eight direction edge response mi is computed for $\mathrm{i}=0,1 \ldots, 7$. LDP code is computed as,

$$
L D P_{k}\left(x_{c}, y_{c}\right)=\sum_{n=0}^{7} 2^{n} * S\left(m_{n}-m_{k}\right), \quad S(x)= \begin{cases}1, & x \geq 0 \\ 0, & x<0\end{cases}
$$

Where $\mathrm{mk}$ is the $\mathrm{k}$-th most significant directional response. Edge response is more stable than the intensity value, sometimes LDP generates same code even though the LBP code is different.

\section{$4 \quad$ Local Mean Binary Pattern}

LBP is simple and efficient but it is sensitive to noise and non-monotonic gray level changes. LDP uses fixed eight masks to measure edge response, so it cannot be stretched to operate at multi-scale or multiresolution. In addition, computing responses in eight directions for each pixel is also computationally intensive. Both the issues, robustness and computation cost are handled by LMBP operator with ease. It assigns an eight-bit binary code to each pixel, which represents the local structure. Multiscale modelling of LMBP is very similar to that of LBP. It may with ease be improved to work at different scale and resolution. In proposed LMBP, the mean value of $3 \times 3$ neighbourhood is compared with eight neighbour values. LMBP code of center pixel $\left(\mathrm{x}_{\mathrm{c}}, \mathrm{y}_{\mathrm{c}}\right)$ is computed as follow:

$$
\operatorname{LMBP}\left(x_{c}, y_{c}\right)=\sum_{n=0}^{7} 2^{n} * S\left(i_{n}-i_{m}\right), \quad S(x)= \begin{cases}1, & x \geq 0 \\ 0, & x<0\end{cases}
$$

Where, $i_{n}$ is the intensity of neighbour of center pixel $\left(\mathrm{x}_{\mathrm{c}}, \mathrm{y}_{\mathrm{c}}\right)$, and $i_{m}$ is the mean intensity of $3 \times 3$ image patch. LMBP of code is resilient to noise and illumination compared to normal LBP operator. The mean of patch suppresses the effect of both, noise and illumination, by diluting the intensity variation. And still, the structural information of the original image is preserved. It provides the stability to generated LMBP code. Working of basic LMBP operator is portrayed in Figure 5. 


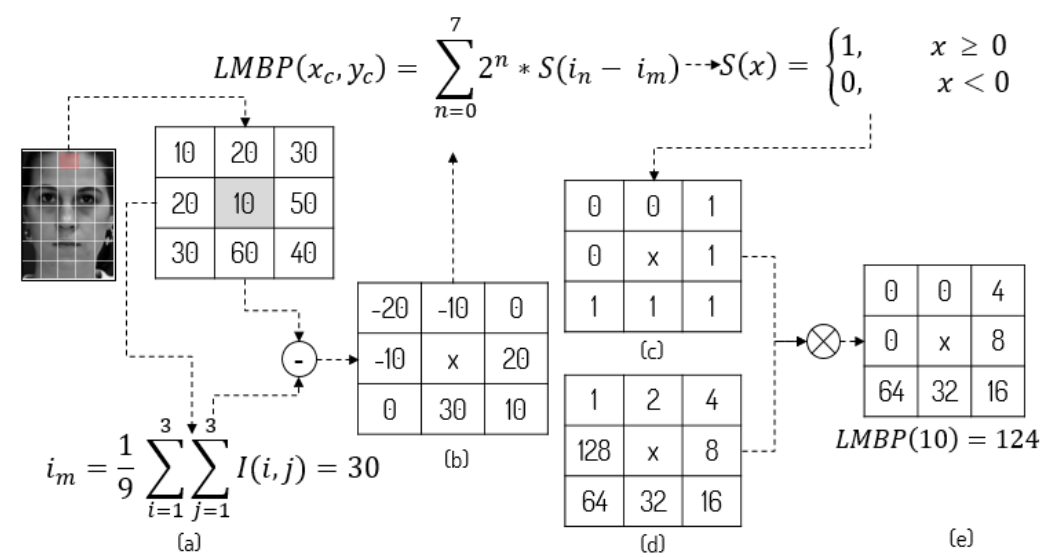

Fig. 5. The procedure of computing LMBP code: (a). Compute mean of $3 \times 3$ patches, (b). Find mean centered patch, (c). The thresholded output of (b), (d). Weight matrix to be applied to (c), (e). Final masked patch used to derive LHMBP value.

The mean value of the patch is subtracted from eight neighbours (Figure 5(b)). Non-negative neighbours are assigned bit 1 , and negative neighbours are assigned bit 0 (Figure 5(c)). The weight matrix shown in Figure 5(d) is applied to thresholded patch. Summation of masked output is the LMBP code of center pixel.

\subsection{Robustness of LMBP}

LMBP binarizes the neighbour pixel with respect to mean value of the patch, and hence it is less susceptible to noise and non-monotonic gray level changes. Thus, robustness is inherent in LMBP operator. Patches in Figure 6(b), Figure 6(c) and Figure 6(d) show the intensity after adding gaussian noise of zero mean and 0.01 variance, zero mean gaussian white noise of local variance 0.5 and multiplicative speckle noise of zero mean and variance 0.1 , respectively. Results show that LBP and LDP codes are sensitive to these noises but LHMBP suppresses the effect of noise, and hence LHMBP code remains unaltered in all three cases.

\begin{tabular}{c|c|c|}
\hline 55 & 31 & 50 \\
\hline 29 & 30 & 30 \\
\hline 20 & 50 & 30 \\
\hline \multicolumn{2}{|c|}{ LBP $=63$} \\
LDP $=253$ \\
LMBP $=37$
\end{tabular}

(a)

\begin{tabular}{|l|l|l|}
\hline 56 & 32 & 50 \\
\hline 30 & 31 & 30 \\
\hline 21 & 51 & 31 \\
\hline \multicolumn{2}{|c|}{$\mathrm{LBP}=55$} \\
$\mathrm{LDP}=125$ \\
$\mathrm{LMBP}=37$
\end{tabular}

(b)

\begin{tabular}{|l|l|l|}
\hline 56 & 32 & 50 \\
\hline 30 & 31 & 30 \\
\hline 21 & 51 & 30 \\
\hline \multicolumn{2}{|c|}{$\mathrm{LBP}=39$} \\
$\mathrm{LDP}=125$ \\
$\mathrm{LMBP}=37$
\end{tabular}

(c)

\begin{tabular}{|l|l|l|}
\hline 55 & 31 & 50 \\
\hline 30 & 30 & 30 \\
\hline 20 & 51 & 31 \\
\hline \multicolumn{3}{|c|}{$\mathrm{LBP}=191$} \\
$\mathrm{LDP}=127$ \\
$\mathrm{LMBP}=37$
\end{tabular}

(d)

Fig. 6. Robustness of LMBP versus. LBP and LDP: (a). LMBP, LBP, and LDP value of original patch. Noisy patch and its LMBP, LBP, and LDP values after adding (b). Gaussian noise, (c). Local variance noise and (d). Speckle noise

LDP generates the feature descriptor by considering the edge response using Kirsch masks, so it is more robust compared to LBP. But due to fixed mask, LDP cannot be extended for larger neighbourhood. Like LBP, an extension of LMBP for the larger neighbourhood is inherent. LDP weights five pixels by -3 and three pixels by 5 , so small change in intensity brings larger change in mask response. Whereas LMBP averages the intensity of all nine pixels, and hence robustness of LMBP is guaranteed over LDP in most of the cases.

\subsection{LMBP Feature Descriptor}

LMBP operator is applied to each pixel of the image. Feature descriptor is obtained by computing the 256 bin histogram of LMBP codes. LMBP histogram is computed as,

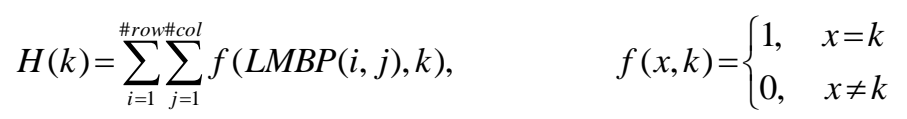

Where $\mathrm{k}$ is the value of LMBP code. Histogram derived using above formula is used as a feature descriptor. Like LBP, LMBP also captures the location independent micro details like edge, spot, and other texture features. While encoding the whole face using LMBP, the global position of such local texture is 
encoded but the precise location is unknown. For the texture analysis, the relation between features and position also plays a vital role. To acquire local features, we divide the image into $\mathrm{M} \times \mathrm{N}$ small regions and 256-bin local histogram $\mathrm{LMBP}^{\mathrm{i}}$ of each region $\mathrm{R}^{\mathrm{i}}$ is computed. All LMBP ${ }^{\mathrm{i}}$ histograms are concatenated to derive the final LMBP descriptor of the whole face. Local histogram corresponds to local details, i.e. texture. Histogram of the whole face encodes the global shape. To incorporate texture and shape both, as shown in Figure 7, we append global histogram of face $\mathrm{LMBP}^{\mathrm{G}}$ to the texture histograms. This feature vector gives precise features for the expression, in which local histograms represent finer detail and global histogram represents coarser detail.

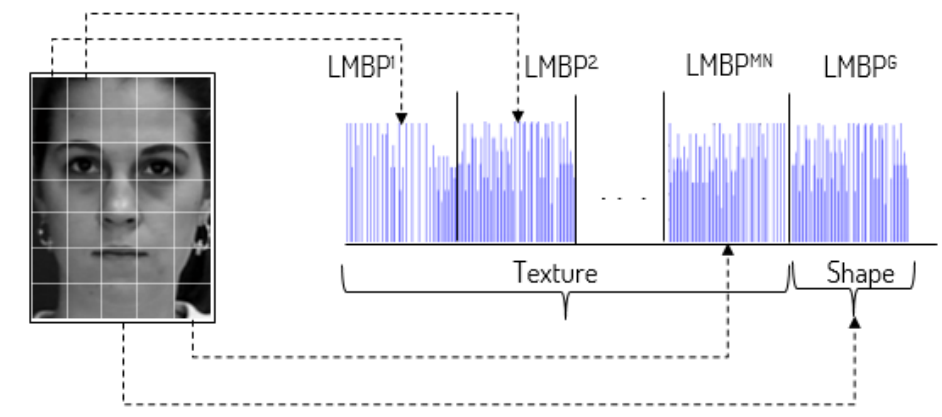

Fig. 7. LMBP descriptor is derived by concatenating local and global histograms of the image.

\section{Experimental Setup}

\subsection{Histogram Normalized Absolute Difference}

Over the time, various classifiers have been proposed to effectively classify the patterns. Chi-square distance, nearest neighbour, neural network, support vector machines are few of them. Comparative analysis of various classifiers for facial expression recognition is discussed in [30]. In our work, we propose a novel distance measure Histogram Normalized Absolute Difference (HNAD). HNAD normalizes the absolute difference of histogram by the histogram union. HNAD distance between two k-bin histograms X and Y is computed as,

$$
\operatorname{HNAD}(X, Y)=\sum_{k} \frac{a b s(X(k)-Y(k))}{\max \{X(k), Y(k)\}}
$$

Computation of HNAD does not involve squaring or square root operations like Chi-square and L2 norm. HNAD is computationally simple yet very effective. Results of all three measures on different datasets are compared in Section 6. Rigorous experiments on different histogram patterns show that HNAD outperforms commonly used measures L2 norm and Chi-square.

\subsection{Experimental Data}

Experiments are conducted on three widely used comprehensive datasets, Cohn-Kanade (CK) [34], Japanese Female Facial Expression (JAFFE) [35] and Taiwanese Facial Expression Image Database (TFEID) [36]. CK dataset contains image sequence of 97 university students having a 7:13 Male: Female ratio. The dataset contains people of different ethnicity, of age group 18-30 years. Each subject was instructed to perform series of 23 facial displays, six of them were based on prototypical expression description. Each image sequence starts with a neutral expression and ends at the apex level.

JAFFE database was planned and assembled by Lyons et al. [35] in 1998. This database is primarily used to evaluate the facial expression recognition systems. It may also be used for face recognition. JAFFE contains 213 images of Ten Japanese female, with 3 or 4 examples of each of the seven basic expressions. Numbers of images corresponding to each of the seven expressions are roughly identical.

TFEID was designed by Chen and Yen at Brain Mapping Laboratory, Taiwan in 2007. It consists of 40 subjects from the same ethnicity with an equal proportion of male and female. Subjects in TFEID are 
instructed to perform eight facial expressions: neutral, anger, contempt, disgust, fear, happiness, sadness and surprise. Models were asked to gaze at two different angles $\left(0^{\circ}\right.$ and $\left.45^{\circ}\right)$. Details of number of images used for the experiment from all datasets are listed in Table 1.

Table 1. Number of images used for the experiment from each dataset.

\begin{tabular}{|l|c|c|c|c|c|c|c|c|}
\hline & AN & DI & FE & HA & SU & SA & NE & Total \\
\hline CK & 110 & 120 & 100 & 280 & 130 & 220 & 320 & 1280 \\
\hline JAFFE & 30 & 29 & 32 & 31 & 31 & 30 & 30 & 213 \\
\hline TFEID & 34 & 40 & 40 & 40 & 39 & 36 & 39 & 268 \\
\hline
\end{tabular}

We considered basic seven expressions anger (AN), disgust (DI), fear (FE), happy (HA), sad (SA), surprise (SU) and neutral (NE), for our problem. Few of the subject from all three datasets with all seven expressions are shown in Figure 8.

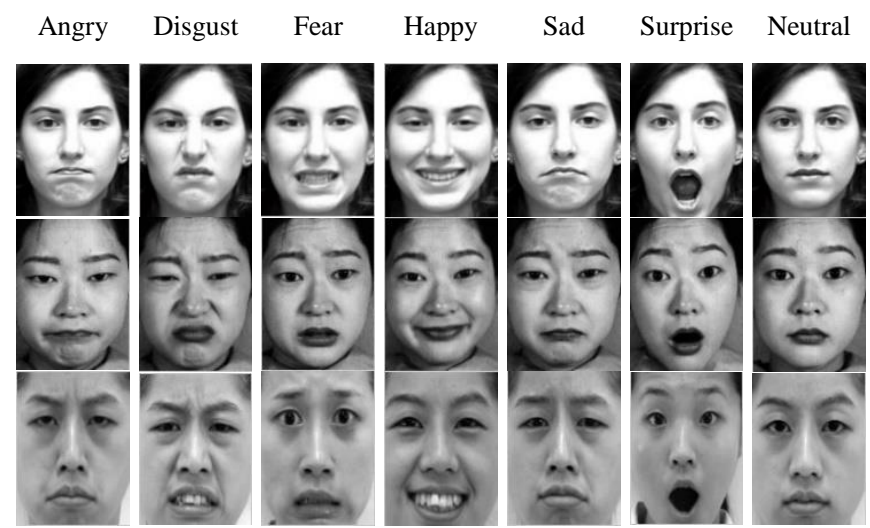

Fig. 8. Snapshots of happy expression from CK (top), JAFFE (middle), and TFEID (bottom) datasets

Researchers have suggested various validation techniques for generalization of classifier. Most widely used validation methods are k-fold validation, Repeated Random Sub-Sampling (RRSS) and Leave One Out (LOO) strategy. In k-fold validation, the dataset is partitioned into k roughly equal folds, of which $\mathrm{k}-1$ folds are used for training and remaining 1-fold is used for testing. In our experiment, 10-fold cross validation strategy is used with seven and six classes, that is with and without neutral expression being a part of the dataset. Results are averaged over 10 executions. Each time dataset is divided into 10 random partitions, out of which 9 are used for training and 1 for testing.

\section{$6 \quad$ Results and Discussion}

The performance of the proposed technique is evaluated for CK, JAFFE, and TFEID dataset using template matching and LS-SVM [37]. In following section, we discussed how optimal parameters are chosen for result analysis.

\subsection{Optimal Parameter Selection}

Single histogram of entire face estimates the global shape of the face. In the prototypic facial expression, textures such as wrinkles, bulges, furs play a crucial role. To extract the local texture features, we divide the face image into $\mathrm{M} \times \mathrm{N}$ regions. 256 bin local LMBP histogram of each region and a global histogram of the whole face are concatenated, which produce the feature vector of size $256^{*}(\mathrm{MN}+1)$.

To find the optimal number of regions, following Jabid et al. [24], Shan et al. [30], and Bartlett et al. [38] we divide images into $3 \times 3,5 \times 5,7 \times 6$ and $9 \times 8$ blocks. Larger image regions fail to capture small size texture leading to poor recognition rate. Whereas, smaller image regions/blocks effectively capture the texture and spatial relationship. However, after a certain point, the smaller size of regions introduces unnecessary computation and feature vector becomes too big to efficiently train the classifier. Performance comparison for a different number of blocks is stated in Table 2. Accuracy is measured using L2 norm, Chi-square 
measure and the proposed distance measure HNAD. The results show that HNAD has better discrimination capacity compared to L2 norm and Chi-square distance. We chose a number of regions to be $5 \times 5$, as it gives a proper balance between accuracy and standard deviation of results from its mean.

Table 2. Recognition rate for a different number of regions, Dataset: CK.

\begin{tabular}{|c|c|c|c|c|c|c|}
\hline & \multicolumn{3}{|c|}{ Accuracy for 7-class problem } & \multicolumn{3}{c|}{ Accuracy for 6-class problem } \\
\hline \#Blocks & L2 Norm & Chi - Square & HNAD & L2 Norm & Chi - Square & HNAD \\
\hline $3 \times 3$ & $95.3 \pm 2.7$ & $95.5 \pm 2.4$ & $96.1 \pm 2.6$ & $98.2 \pm 1.9$ & $98.1 \pm 2.1$ & $98.0 \pm 2.2$ \\
\hline $5 \times 5$ & $94.1 \pm 2.1$ & $94.5 \pm 2.1$ & $95.8 \pm 1.8$ & $98.3 \pm 1.3$ & $98.3 \pm 1.3$ & $98.4 \pm 1.4$ \\
\hline $7 \times 6$ & $95.2 \pm 1.8$ & $95.2 \pm 1.8$ & $96.9 \pm 1.7$ & $98.1 \pm 2.5$ & $98.0 \pm 2.4$ & $98.1 \pm 2.5$ \\
\hline $9 \times 8$ & $94.9 \pm 2.2$ & $95.4 \pm 1.5$ & $96.3 \pm 1.5$ & $99.2 \pm 1.1$ & $99.2 \pm 1.1$ & $99.3 \pm 1.1$ \\
\hline
\end{tabular}

\subsection{Performance Evaluation using Optimal Parameter Selection}

From experimental results, we chose an optimal number of blocks to be $5 \times 5$. Using HNAD measure, we achieved a recognition rate of $95.8 \%$ and $98.4 \%$ for a 7 -class and 6-class problem respectively for CK dataset. Similarly, the accuracy of $97.2 \%$ and $95.2 \%$ is reported on JAFFE for a 6-class and 7-class problems. Comparison of proposed method with various local descriptors like Gabor [38], LBP [30] and LDP [24] is shown in Table 3 for CK and JAFFE dataset. Results show the superiority of proposed method with HNAD measure.

Table 3. Performance comparison on CK and JAFFE dataset using template matching.

\begin{tabular}{|l|c|c|c|c|}
\hline & \multicolumn{2}{|c|}{ CK } & \multicolumn{2}{c|}{ JAFFE } \\
\hline Descriptor & $\mathrm{C}=6$ & $\mathrm{C}=7$ & $\mathrm{C}=6$ & $\mathrm{C}=7$ \\
\hline Gabor [38] & $83.7 \pm 4.5$ & $78.9 \pm 4.8$ & $81.9 \pm 6.4$ & $75.5 \pm 5.8$ \\
\hline LBP [30] & $84.5 \pm 5.2$ & $79.1 \pm 4.6$ & $83.7 \pm 6.7$ & $77.2 \pm 7.6$ \\
\hline LDP [24] & $89.2 \pm 2.5$ & $86.9 \pm 2.8$ & $87.4 \pm 5.6$ & $82.6 \pm 4.1$ \\
\hline LMBP & $\mathbf{9 8 . 4} \pm \mathbf{1 . 4}$ & $\mathbf{9 5 . 8} \pm \mathbf{1 . 8}$ & $\mathbf{9 5 . 2} \pm \mathbf{2 . 2}$ & $\mathbf{9 7 . 2} \pm \mathbf{1 . 7}$ \\
\hline
\end{tabular}

In recent time, LS-SVM [37] has emerged as a first choice classifier for researchers. We also conduct experiments using LS-SVM with different kernels. Block based approach produces a very big feature vector. For $\mathrm{M} \times \mathrm{N}$ blocks, LMBP produces the feature vector of size $256 *(\mathrm{MN}+1)$. Training the classifier for such a big vector is very difficult and time-consuming. Huge feature vector often leads to over trained model. In order to reduce the dimensions of the feature vector, we used principal component analysis. From experiment, we chose first 70 eigenvectors with highest eigenvalue for the projection. Results of this experiment for state of the art methods on CK dataset are compared in Table 4. With RBF kernel, we achieved highest recognition rate among all compared methods.

Table 4. Performance comparison of different methods on CK dataset using support vector machine

\begin{tabular}{|l|c|c|c|c|c|c|}
\hline & \multicolumn{3}{|c|}{ Accuracy for 6-class problem } & \multicolumn{3}{c|}{ Accuracy for 7-class problem } \\
\hline Technique & Linear (\%) & Poly. (\%) & RBF (\%) & Linear (\%) & Poly. (\%) & RBF (\%) \\
\hline Gabor [38] & $89.4 \pm 3.0$ & $89.4 \pm 3.0$ & $89.8 \pm 3.1$ & $86.6 \pm 4.1$ & $86.6 \pm 4.1$ & $86.8 \pm 3.6$ \\
\hline LBP [30] & $91.5 \pm 3.1$ & $91.5 \pm 3.1$ & $92.6 \pm 2.9$ & $88.1 \pm 3.8$ & $88.1 \pm 3.8$ & $88.9 \pm 3.5$ \\
\hline LDP [24] & $94.9 \pm 1.2$ & $94.9 \pm 1.2$ & $96.4 \pm 0.9$ & $92.8 \pm 1.7$ & $92.8 \pm 1.7$ & $93.4 \pm 1.5$ \\
\hline LMBP & $\mathbf{9 2 . 1} \pm \mathbf{2 . 9}$ & $\mathbf{9 8 . 9} \pm \mathbf{1 . 7}$ & $\mathbf{9 9 . 3} \pm \mathbf{1 . 4}$ & $\mathbf{8 6 . 6} \pm \mathbf{2 . 8}$ & $\mathbf{9 7 . 8} \pm \mathbf{1 . 5}$ & $\mathbf{9 8 . 2} \pm \mathbf{1 . 0}$ \\
\hline
\end{tabular}

A similar experiment is conducted on JAFFE dataset. Comparison of proposed method with existing work on JAFFE dataset is presented in Table 5. 
Table 5. Performance comparison of different methods on JAFFE using support vector machine

\begin{tabular}{|l|c|c|c|c|c|c|}
\hline & \multicolumn{3}{|c|}{ Accuracy for 6-class problem } & \multicolumn{3}{c|}{ Accuracy for 7-class problem } \\
\hline Technique & Linear (\%) & Poly. (\%) & RBF (\%) & Linear (\%) & Poly. (\%) & RBF (\%) \\
\hline Gabor [38] & $85.1 \pm 5.0$ & $85.1 \pm 5.0$ & $85.8 \pm 4.1$ & $79.7 \pm 4.2$ & $79.7 \pm 4.2$ & $80.8 \pm 3.7$ \\
\hline LBP [30] & $86.7 \pm 4.1$ & $86.7 \pm 4.1$ & $87.5 \pm 5.1$ & $80.7 \pm 5.5$ & $80.7 \pm 5.5$ & $81.9 \pm 5.2$ \\
\hline LDP [24] & $89.9 \pm 5.2$ & $89.9 \pm 5.2$ & $90.1 \pm 4.9$ & $84.9 \pm 4.7$ & $84.9 \pm 4.7$ & $85.4 \pm 4.0$ \\
\hline LMBP & $\mathbf{8 7 . 8} \pm \mathbf{2 . 2}$ & $\mathbf{9 1 . 2} \pm \mathbf{2 . 1}$ & $\mathbf{9 2 . 3} \pm \mathbf{2 . 5}$ & $\mathbf{8 1 . 7} \pm \mathbf{3 . 2}$ & $\mathbf{8 0 . 6} \pm \mathbf{2 . 2}$ & $\mathbf{8 8 . 3} \pm \mathbf{1 . 4}$ \\
\hline
\end{tabular}

TFEID dataset is not much explored by researchers. We test the robustness of our method on TFEID also, and results are depicted in Table 6. As stated earlier, we chose 70 features after PCA projection. Optimal number of blocks chosen for experiment are also same as earlier, i.e. $5 \times 5$.

Table 6. Performance of LMBP on TFEID

\begin{tabular}{|c|c|c|c|c|c|c|}
\hline & \multicolumn{3}{|c|}{ Template Matching } & \multicolumn{3}{c|}{ Machine Learning } \\
\hline \#Class & L2 Norm & Chi - Square & HNAD & Linear (\%) & Polynomial (\%) & RBF (\%) \\
\hline 6 & $89.9 \pm 4.1$ & $90.31 \pm 4.3$ & $91.78 \pm 3.9$ & $92.9 \pm 4.1$ & $85.8 \pm 3.6$ & $92.5 \pm 4.2$ \\
\hline 7 & $86.4 \pm 5.2$ & $86.5 \pm 5.3$ & $89.9 \pm 5.2$ & $90.0 \pm 4.8$ & $87.9 \pm 5.6$ & $90.4 \pm 5.1$ \\
\hline
\end{tabular}

Results discussed so far were averaged over all six or seven expressions. To get a better idea about recognition rate of individual expression, confusion matrix for CK, JAFFE, and TFEID are shown in Table 7. Results are reported using LS-SVM with RBF kernel.

Table 7. Confusion matrix for a 7-class problem for all three datasets, classifier: LS-SVM (RBF)

\begin{tabular}{|c|c|c|c|c|c|c|c|c|c|c|c|c|c|c|c|c|c|c|c|c|c|}
\hline & \multicolumn{7}{|c|}{ Confusion matrix for CK } & \multicolumn{7}{|c|}{ Confusion matrix for JAFFE } & \multicolumn{7}{|c|}{ Confusion matrix for TFEID } \\
\hline & $\mathbf{A N}$ & DI & FE & HA & SA & SU & NE & AN & DI & FE & HA & SA & SU & $\mathrm{NE}$ & AN & DI & FE & HA & SA & SU & $\mathbf{N E}$ \\
\hline $\mathbf{A N}$ & 98.0 & 0.3 & 0.7 & 0.0 & 0.8 & 0.0 & 0.2 & 85.6 & 2.3 & 5.3 & 1.1 & 3.3 & 0.8 & 1.6 & 88.3 & 5.2 & 2.3 & 1.0 & 1.5 & 0.6 & 1.1 \\
\hline DI & 0.6 & 97.4 & 0.8 & 0.1 & 0.9 & 0.0 & 0.2 & 4.6 & 84.4 & 4.8 & 0.7 & 3.1 & 0.5 & 1.9 & 4.5 & 87.2 & 3.6 & 1.4 & 1.1 & 0.9 & 1.3 \\
\hline FE & 0.8 & 0.8 & 97.7 & 0.1 & 0.1 & 0.3 & 0.2 & 5.6 & 3.4 & 85.2 & 1.6 & 2.1 & 0.0 & 2.1 & 3.2 & 2.1 & 91.1 & 0.6 & 1.3 & 0.8 & 0.9 \\
\hline HA & 0.1 & 0.1 & 0.1 & 99.6 & 0.1 & 0.0 & 0.0 & 2.1 & 1.2 & 1.1 & 93.2 & 0.7 & 0.3 & 1.4 & 1.4 & 1.1 & 2.9 & 92.1 & \begin{tabular}{ll|}
0.4 \\
\end{tabular} & $\begin{array}{ll}1.1 \\
\end{array}$ & \begin{tabular}{ll|}
1.0 \\
\end{tabular} \\
\hline SA & 0.7 & 1.1 & 0.6 & 0.2 & 96.2 & 0.2 & 1.0 & 2.3 & 3.8 & 2.3 & 1.7 & 85.2 & 1.2 & 3.5 & $\begin{array}{ll}1.9 \\
\end{array}$ & 2.1 & 3.4 & \begin{tabular}{|l|l}
1.1 \\
\end{tabular} & 89.2 & \begin{tabular}{|l|l}
0.2 \\
\end{tabular} & 2.1 \\
\hline SU & 0.0 & 0.1 & 0.1 & 0.0 & 0.0 & 99.8 & 0.0 & 0.9 & 1.7 & 2.3 & 1.1 & 0.7 & 93.2 & 0.1 & 1.2 & 0.7 & 2.1 & 0.8 & 0.1 & 94.2 & 0.9 \\
\hline $\mathbf{N E}$ & $\begin{array}{l}0.1 \\
\end{array}$ & 0.2 & 0.3 & 0.2 & 0.4 & 0.1 & 98.7 & 1.9 & 0.9 & 1.5 & 0.8 & 3.5 & 0.2 & 91.2 & $\begin{array}{ll}0.7 \\
\end{array}$ & $\begin{array}{ll}1.8 \\
\end{array}$ & 1.4 & 0.6 & 4.2 & \begin{tabular}{|l|l}
0.3 \\
\end{tabular} & 91.0 \\
\hline Avg. & \multicolumn{7}{|c|}{98.2} & \multicolumn{7}{|c|}{88.3} & \multicolumn{7}{|c|}{90.4} \\
\hline
\end{tabular}

For CK dataset, the average accuracy of $98.2 \%$ for seven classes is really encouraging, compared to 91.4\% accuracy reported by Shan et al [30]. Bartlett et al. [38] obtained the highest recognition rate of 93.3\% using a subset of Gabor filters with Adaboost feature selection and SVM classifier. Using three layers neural network, Tian [39] achieved 94\% recognition rate with Gabor features along with geometric features. Results of proposed method are compared with various existing approaches on CK dataset in Table 8.

Table 8. Reported accuracy of state of the art methods on CK dataset.

\begin{tabular}{|l|c|c|c|c|}
\hline Literature & \#Subjects & \#Class & Strategy & Acc.(\%) \\
\hline Yang et al. [40] & 96 & 6 & - & 92.3 \\
\hline Shan et al. [30] & 96 & 6 & 10 Fold & 92.1 \\
\hline Littlewort et al. [41] & 90 & 7 & - & 93.3 \\
\hline Wenfei et al. [42] & 94 & 7 & 10 Fold & 91.5 \\
\hline Oyuang et al. [43] & 94 & 6 & - & 93.5 \\
\hline
\end{tabular}




\begin{tabular}{|l|c|c|c|c|}
\hline Literature & \#Subjects & \#Class & Strategy & Acc.(\%) \\
\hline Zheng et al. [44] & 97 & 6 & 10 -Fold & 94.0 \\
\hline LMBP + HNAD & 97 & 7 & 10 -Fold & $\mathbf{9 5 . 8}$ \\
\hline LMBP + HNAD & 97 & 6 & 10 -Fold & $\mathbf{9 8 . 4}$ \\
\hline LMBP + SVM & 97 & 7 & 10 -Fold & $\mathbf{9 8 . 2}$ \\
\hline LMBP + SVM & 97 & 6 & 10 -Fold & $\mathbf{9 9 . 3}$ \\
\hline
\end{tabular}

JAFFE dataset is another widely accepted facial expression dataset, developed by Lyons et al [25]. JAFFE does not have as much diversity as CK has. Subjects in JAFFE belong to the same ethnicity and all are female. The highest recognition rate of $88.3 \%$ and $92.3 \%$ is achieved using SVM with RBF kernel for seven and six class problem, respectively. Results of proposed method are compared with various state of the art methods in Table 9 for JAFFE dataset.

Table 9. Reported accuracy of state of the art methods on JAFFE dataset.

\begin{tabular}{|l|c|c|}
\hline Literature & Method & Acc. (\%) \\
\hline Lekshmi and Sasikumar [45] & SVM & 86.9 \\
\hline Zhi and Ruan [46] & 2D-DLPP & 95.9 \\
\hline Zhao, Zhuang, and Xu [47] & PCA + ANN & 93.7 \\
\hline Owusu et al. [48] & Gabor + MFFNN & 96.8 \\
\hline \multirow{4}{*}{ Proposed } & LMBP + HNAD (C =7) & $\mathbf{9 7 . 2}$ \\
\cline { 2 - 3 } & LMBP + HNAD (C =6) & $\mathbf{9 5 . 2}$ \\
\cline { 2 - 3 } & LMBP + SVM (C =7) & $\mathbf{8 8 . 3}$ \\
\cline { 2 - 3 } & LMBP + SVM (C =6) & $\mathbf{9 2 . 3}$ \\
\hline
\end{tabular}

\subsection{Expression Recognition in Low Resolution}

It is not always possible to acquire high-quality images under all circumstances. In certain applications such as home monitoring, surveillance applications, smart meeting, only low-resolution videos are available [39]. Expression recognition in low resolution is an almost unaddressed area, very little work is done in this field. In our experiment, we studied the performance of LMBP operator at four different resolutions: $150 \times$ $110,75 \times 55,48 \times 36$ and $37 \times 27$. Low-resolution images are derived by down-sampling the original images. Shan et al. [30] conducted the experiment in low resolution with LBP and Gabor features derived by convolving the image with 40 gabor filter banks. The classification was performed using SVM. Tian [39] selected 375 image sequences from CK dataset. He used geometric and appearance features. Geometric features were computed using feature tracking and feature selection. Appearance features were derived using 40 gabor filter banks. The three-layer neural network was used for expression classification. Due to down sampling and smaller size of the image, geometric features do not make sense, as detecting and tracking geometric feature is also difficult and vulnerable in such environment. So, only appearance features should be considered for classification. Jabid et al. [24] derived LDP features using 8 Kirsch edge detectors. They achieved notable recognition rate using SVM. In Table 10, the performance of LMBP in low resolution is compared with various existing approaches. 
Table 10. Performance comparison of different methods in low-resolution environment

\begin{tabular}{|c|c|c|c|c|}
\hline Resolution & $150 \times 110$ & $75 \times 55$ & $48 \times 36$ & $37 \times 27$ \\
\hline \multicolumn{5}{|c|}{ Feature Descriptor } \\
\hline Gabor [39] & 92.2 & 91.6 & - & 77.6 \\
\hline Gabor [38] & $89.1 \pm 3.1$ & $89.2 \pm 3.0$ & $86.4 \pm 3.3$ & $83.0 \pm 4.3$ \\
\hline LBP [30] & $92.6 \pm 2.9$ & $89.9 \pm 3.1$ & $87.3 \pm 3.4$ & $84.3 \pm 4.1$ \\
\hline Gabor [30] & $89.8 \pm 3.1$ & $89.2 \pm 3.0$ & $86.4 \pm 3.3$ & $83.0 \pm 4.3$ \\
\hline LDP [24] & $96.4 \pm 0.9$ & $95.5 \pm 1.6$ & $93.1 \pm 2.2$ & $90.6 \pm 2.7$ \\
\hline LMBP & $95.9 \pm 1.7$ & $97.0 \pm 1.8$ & $95.9 \pm 2.9$ & $94.8 \pm 1.7$ \\
\hline
\end{tabular}

\section{Conclusions and Future Work}

In this paper, we present a novel facial expression descriptor, Local Mean Binary Pattern and a novel classifier Histogram Normalized Absolute Difference. Gabor involves extensive computation due to the convolution of image with a large filter bank. LBP is sensitive to both, noise and non-monotonic gray level change. LDP is also computationally expensive as it requires eight masks to compute a single LDP code. As LMBP performs the thresholding with respect to mean of the patch, it can sustain against noise and illumination change stronger than other descriptors. LMBP is simple and fast yet quite effective.

The performance of LMBP is evaluated in the different scenario for CK, JAFFE, and TFEID. Results confirm the consistency of LMBP in the sense that it achieves good recognition rate with very less standard deviation. In an uncompressed domain, with HNAD proposed method achieves a recognition rate of $98.4 \% \pm$ 1.4 and $95.8 \% \pm 1.8$ for CK dataset, and for JAFFE it achieves $95.2 \% \pm 2.2$ and $97.2 \% \pm 1.7$ for 6 -class and 7-class problem, respectively. Even in the low-resolution environment, LMBP achieves a recognition rate of $94.8 \%$ for $37 \times 27$ image on $\mathrm{CK}$, which is remarkable.

In future, LMBP operator may be extended for temporal domain. Rotation invariance property of LMBP operator is yet to be explored. Like LBP, uniform pattern shall be derived and tested for LMBP too.

\section{References}

[1] A. Mehrabian, "Communication without words," Psychol. Today, vol. 2, pp. 53-55, 1968.

[2] D. Freire, "Smile detection using Local Binary Pattern and Support Vector Machine," in 4th International Conference on Computer Vision Theory and Applications, 2002.

[3] J. Whitehill, G. Littlewort, I. Fasel, M. Bartlett, and J. Movellan, "Toward Practical Smile Detection," IEEE Trans. Pattern Anal. Mach. Intell., vol. 31, no. 11, pp. 2106-2111, 2009. DOI: http://dx.doi.org/10.1109/TPAMI.2009.42

[4] C. Shan, "Smile Detection by Boosting Pixel Differences," IEEE Trans. image Process., vol. 21, no. 1, pp. 431-436, 2012.

[5] Q. Ji, Z. Zhu, and P. Lan, "Real-time nonintrusive monitoring and prediction of driver fatigue," IEEE Trans. Veh. Technol., vol. 53, no. 4, pp. 1052-1068, 2004. DOI: http://dx.doi.org/10.1109/TVT.2004.830974

[6] B. Gholami, W. M. Haddad, and A. R. Tannenbaum, "Relevance Vector Machine Learning for Neonate Pain Intensity Assessment Using Digital Imaging," IEEE Trans. Biomed. Eng., vol. 57, no. 6, pp. 1457-1466, 2010. DOI: http://dx.doi.org/10.1109/TBME.2009.2039214 
[7] B. Fasel and J. Luettin, "Automatic facial expression analysis: A survey," Pattern Recognit., vol. 36, no. 1, pp. 259-275, 2003. DOI: http://dx.doi.org/10.1016/S0031-3203(02)00052-3

[8] Z. Zeng, M. Pantic, G. I. Roisman, and T. S. Huang, "A survey of affect recognition methods: Audio, visual, and spontaneous expressions," IEEE Trans. Pattern Anal. Mach. Intell., vol. 31, no. 1, pp. 39 58, 2009. DOI: http://dx.doi.org/10.1109/TPAMI.2008.52

[9] M. Pantic and I. Patras, "Dynamics of facial expression: Recognition of facial actions and their temporal segments from face profile image sequences," IEEE Trans. Syst. Man, Cybern. Part B Cybern., vol. 36, no. 2, pp. 433-449, 2006. DOI: http://dx.doi.org/10.1109/TSMCB.2005.859075

[10] T. R. Almaev and M. F. Valstar, "Local Gabor Binary Patterns from three orthogonal planes for automatic facial expression recognition," in Humaine Association Conference on Affective Computing and Intelligent Interaction, 2013, pp. 356-361. DOI: http://dx.doi.org/10.1109/ACII.2013.65

[11] R. Highfield, R. Wiseman, and R. Jenkins, "How your looks betray personality," New Scientists, 2009.

[12] C. Darvin, The Expression of the Emotions in Man and Animals. London: J. Murray, 1872.

[13] P. Ekman and W. V Friesen, "Constants across cultures in the face and emotion," J. Pers. Soc. Psychol., vol. 17, no. 2, pp. 124-129, 1971. DOI: http://dx.doi.org/10.1037/h0030377

[14] C. A. Corneanu, O. Marc, J. F. Cohn, and E. Sergio, "Survey on RGB, 3D, Thermal, and Multimodal approaches for facial expression recognition: History, trends, and affect-related applications," IEEE Trans. Pattern Anal. Mach. Intell., vol. 38, no. 8, pp. 1548-1568, 2016. DOI: http://dx.doi.org/10.1109/TPAMI.2016.2515606

[15] Y. Tian, T. Kanade, and J. F. Cohn, "Evaluation of Gabor-wavelet-based facial action unit recognition in image sequences of increasing complexity," in IEEE International Conference on Automatic Face and Gesture Recognition, 2002, pp. 2-7.

[16] T. Ojala, P. Matti, and H. David, "A comparative study of texture measures with classification based on featured distributions," Pattern Recognit., vol. 29, no. 1, pp. 51-59, 1996. DOI: http://dx.doi.org/10.1016/0031-3203(95)00067-4

[17] Y. Yacoob and L. S. Davis, "Recognizing human facial expressions from long image sequences using Optical Flow," IEEE Trans. Pattern Anal. Mach. Intell., vol. 18, no. 6, pp. 636-642, 1996. DOI: http://dx.doi.org/10.1109/34.506414

[18] M. Rosenblum, Y. Yacoob, and L. S. Davis, "Human expression recognition from motion using a radial basis function network architecture.," IEEE Trans. Neural Networks, vol. 7, no. 5, pp. 11211138, 1996. DOI: http://dx.doi.org/10.1109/72.536309

[19] M. A. Turk and A. P. Pentland, "Face recognition using eigenfaces," Journal of Cognitive Neuroscience, vol. 3, no. 1. pp. 72-86, 1991. DOI: http://dx.doi.org/10.1109/CVPR.1991.139758

[20] P. N. Belhumeur, J. P. Hespanha, and D. J. Kriegman, "Eigenfaces vs. Fisherfaces: Recognition using class specific linear projection," IEEE Trans. Pattern Anal. Mach. Intell., vol. 19, no. 7, pp. 711-720, 1997. DOI: http://dx.doi.org/10.1109/34.598228

[21] M. Bartlett and T. J. Sejnowski, "Independent components of face images: A representation for face recognition," in 4th Annual Joint Symposium on Neural Computation, 1997.

[22] T. Ojala, M. Pietikainen, and D. Harwood, "A comparative study of texture measures with classification based on feature distributions," Pattern Recognit., vol. 29, no. 1, pp. 51-59, 1996.

[23] T. Ojala, M. Pietikäinen, and T. Mäenpää, "A generalized local binary pattern operator for multiresolution gray scale and rotation invariant texture classification," in International Conference on Advances in Pattern Recognition, 2001, pp. 399-408.

[24] T. Jabid, M. H. Kabir, and O. Chae, "Robust facial expression recognition based on local directional pattern," ETRI J., vol. 32, no. 5, pp. 784-794, $2010 . \quad$ DOI: http://dx.doi.org/10.4218/etrij.10.1510.0132

[25] M. Lyons and S. Akamatsu, "Coding facial expressions with gabor wavelets," in third IEEE Conference on Automatic Face and Gesture Recognition, 1998, pp. 200-205. DOI: http://dx.doi.org/10.1109/AFGR.1998.670949 
[26] A. Samal and P. A. Lyengar, "Automatic recognition and analysis of human faces and facial expressions : A survey," IEEE Trans. Pattern Recognit., vol. 25, no. 1, pp. 65-77, 1992.

[27] Y. L. Tian, T. Kanade, and J. F. Cohn, "Facial expression analysis," Handb. Face Recognit., pp. 247275, 2005. DOI: http://dx.doi.org/10.1007/0-387-27257-7_12

[28] M. N. Dailey, C. Joyce, M. J. Lyons, M. Kamachi, H. Ishi, J. Gyoba, and G. W. Cottrell, "Evidence and a computational explanation of cultural differences in facial expression recognition.," Emotion, vol. 10, no. 6, pp. 874-893, 2010. DOI: http://dx.doi.org/10.1037/a0020019

[29] M. Murtaza, M. Sharif, M. Raza, and J. H. Shah, "Analysis of Face Recognition under varying facial expression: A survey,” Int. Arab J. Inf. Technol., vol. 10, no. 5, pp. 378-388, 2013.

[30] C. Shan, S. Gong, and P. W. Mcowan, "Facial expression recognition based on Local Binary Patterns: A comprehensive study," Image Vis. Comput., vol. 27, no. 6, pp. 803-816, 2009.

[31] T. Ojala, M. Pietikainen, and T. Maenpaa, "Multiresolution gray-scale and rotation invariant texture classification with local binary patterns," IEEE Trans. Pattern Anal. Mach. Intell., vol. 24, no. 7, pp. 971-987, 2002. DOI: http://dx.doi.org/10.1109/TPAMI.2002.1017623

[32] T. Senechal, V. Rapp, H. Salam, R. Seguier, K. Bailly, and L. Prevost, "Facial action recognition combining heterogeneous features via multikernel learning," IEEE Trans. Syst. Man Cybern. B Cybern., vol. 42, no. 4, pp. 993-1005, 2012. DOI: http://dx.doi.org/10.1109/TSMCB.2012.2193567

[33] R. Hablani, N. Chaudhari, and S. Tanwani, "Recognition of facial expressions using Local Binary Patterns of important facial parts," Int. J. Image Process., vol. 7, no. 2, pp. 163-170, 2013.

[34] T. Kanade, J. F. Cohn, and Y. Tian, "Comprehensive database for facial expression analysis," in 4th IEEE International Conference on Automatic Face and Gesture Recognition, 2000, pp. 46-53. DOI: http://dx.doi.org/10.1109/AFGR.2000.840611

[35] M. J. Lyons, "Automatic classification of single facial images," IEEE Trans. Pattern Anal. Mach. Intell., vol. 21, no. 12, pp. 1357-1362, 1999. DOI: http://dx.doi.org/10.1109/34.817413

[36] L.-F. Chen and Y.-S. Yen, "Taiwanese Facial Expression Image Database." Brain Mapping Laboratory, Institute of Brain Science, National Yang-Ming University, Taipei, Taiwan., 2007.

[37] J. A. K. Suykens, L. Lukas, P. Van Dooren, B. De Moor, and J. Vandewalle, "Least Squares Support Vector Machine classifers: A large scale algorithm," in European Conference on Circuit Theory and Design, 1999, pp. 839-842.

[38] M. S. Bartlett, G. Littlewort, M. Frank, C. Lainscsek, I. Fasel, and J. Movellan, "Recognizing facial expression: Machine learning and application to spontaneous behavior," in IEEE Computer Society Conference on Computer Vision and Pattern Recognition, 2005, vol. 2, pp. 568-573.

[39] Y. T. Y. Tian, "Evaluation of Face Resolution for Expression Analysis," in 2004 Conference on Computer Vision and Pattern Recognition Workshop, 2004, pp. 1-7.

[40] P. Yang, Q. Liu, and D. N. Metaxas, "Exploring facial expressions with compositional features," in IEEE Computer Society Conference on Computer Vision and Pattern Recognition, 2010, pp. 26382644. DOI: http://dx.doi.org/10.1109/CVPR.2010.5539978

[41] G. Littlewort, M. S. Bartlett, I. Fasel, J. Susskind, and J. Movellan, "Dynamics of facial expression extracted automatically from video," Image Vis. Comput., vol. 24, no. 6, pp. 615-625, 2006. DOI: http://dx.doi.org/10.1016/j.imavis.2005.09.011

[42] G. Wenfei, C. Xiang, Y. V. Venkatesh, D. Huang, and H. Lin, "Facial expression recognition using radial encoding of local Gabor features and classifier synthesis," Pattern Recognit., vol. 45, no. 1, pp. 80-91, 2012.

[43] Y. Ouyang, N. Sang, and R. Huang, "Robust automatic facial expression detection method based on sparse representation plus LBP map," Opt. - Int. J. Light Electron Opt., vol. 124, no. 24, pp. 68276833, 2013. DOI: http://dx.doi.org/10.1016/j.ijleo.2013.05.076

[44] H. Zheng, X. Geng, D. Tao, and Z. Jin, "A Multi-Task Model for simultaneous face identification and facial expression recognition," Neurocomputing, vol. 171, pp. 515-523, 2016. DOI: http://dx.doi.org/10.1016/j.neucom.2015.06.079 
[45] L. Praseeda and Sasikumar, "Analysis of facial expression using Gabor and SVM," Int. J. Recent Trends Eng., vol. 1, no. 2, pp. 47-50, 2009.

[46] R. Zhi and Q. Ruan, "Facial expression recognition based on Two-dimensional Discriminant Locality Preserving Projections," Neurocomputing, vol. 71, no. 7, pp. 1730-1734, 2008. DOI: http://dx.doi.org/10.1016/j.neucom.2007.12.002

[47] L. Zhao, "Facial expression recognition based on PCA and NMF," in 7th World Congress on Intelligent Control and Automation, 2008, no. 4, pp. 6826-6829.

[48] E. Owusu, Y. Zhan, and Q. R. Mao, "A Neural-AdaBoost based facial expression recognition system," Expert Syst. Appl., vol. 41, no. 7, pp. 3383-3390, 2014. DOI: http://dx.doi.org/10.1016/j.eswa.2013.11.041 